\title{
Perceptual Image Watermarking based on a Mixed-scale Wavelet Representation
}

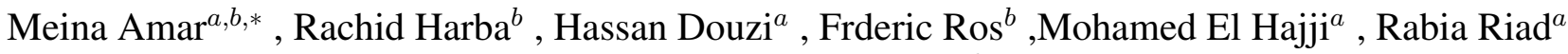 \\ and Khadija Gourrame ${ }^{a, b}$ \\ ${ }^{a}$ IRF-SIC Laboratory Ibn Zohr University, BP 8106 - City Dakhla, 80000 Agadir, Morocco \\ ${ }^{b}$ PRISME Laboratory University of Orlans, 12 Rue de Blois, 45067 Orlans, France
}

\begin{abstract}
Perceptual image watermarking consists in using knowledge of the Human Visual System (HVS) to choose the strength of the watermark according to image properties. This paper proposes a new perceptual image watermarking method that combines the advantages of both the wavelet domain and the spatial domain since a mixed-scale wavelet representation is applied. By considering the density of the dominant wavelet coefficients, our scheme is able to differentiate uniform, edge and texture areas. This allowed us to apply adapted luminance, edge or texture masking more efficiently. We selected effective Just Noticeable Difference models from the literature, i.e. luminance and edge masking developed by Chou and $\mathrm{Li}$, and texture masking developed by Qi et al. We also took into account the HVS sensitivity, which varies with the orientation of the image activity. The method was tested on a large database of 50 color images, and compared with four other watermarking methods from the literature. Visual quality tests were conducted and the robustness to attacks was tested compared with four other watermarking methods from the literature. Results show that the proposed method yields a high visual quality and our method is very robust against attacks. This last point is of great importance for real applications. The proposed method is the best trade-off between visual quality and resistance to attacks among the tested methods.
\end{abstract}

\section{Keywords}

Perceptual models, Human Visual System, digital watermarking, wavelet, lifting scheme

\section{INTRODUCTION}

Human Visual System (HVS) studies have become an important issue in image processing [1. 2]. Many factors cause human vision to have different sensitivity to disturbs or noise with respect to local properties of the image. Barni [3] summarized these observations and experiments for grey scale images in the following four rules:

-Rule 1: disturbs are much less visible on highly textured regions than on uniform areas.

- Rule 2: contours are more sensitive to noise addition than highly textured regions but less sensitive than flat areas.

-Rule 3: disturbs are less visible over dark and bright regions.

-Rule 4: the eye is more sensitive to disturbs on vertical or horizontal high frequency waves than those at 45.
Perceptual watermarking uses these properties to hide the watermark effectively. Watermarking is an efficient technique to guarantee the safety of multimedia documents [4]. The most important objective in digital watermarking is to solve the trade-off between invisibility and robustness [5.6]. Therefore, the aim is to guarantee that the embedded watermark be imperceptible to human eyes and at the same time to ensure that the watermark can be successfully extracted even if the watermarked image is attacked. To improve both watermarked image quality and robustness of the watermark, the most efficient techniques take into account the properties of the HVS [7] by developing perceptual masks. Models based on the HVS determine the strength of the watermark that can be added to each pixel.

Image watermarking is composed of two steps: i) insertion of the mark in an image; ii) extraction of the mark [4, 8]. In psychovisual studies, a level of distortion that can just be seen in experimental tests is called JND (Just Noticeable Difference) [4 9]. The JND is used to adjust the embedding strength, which is optimized and adapted with respect to areas in the image. The principle of digital watermarking using JND in the spatial domain can be described by the following expression:

$$
I_{w}(x, y)=I_{o}(x, y)+J N D(x, y) * W(x, y)
$$

where $I_{w}$ is the watermarked image, and $I_{o}$ the original image. The watermark $W$ is a pseudo-random sequence of $\{+1,-1\}$, and JND is the strength of the watermark.

Several important perceptual masks have been designed in the spatial domain and in transformed domains. In [10] Chou and Li proposed a spatial domain JND model for compression. This model takes into account the luminance adaptation and a so-called activity masking. The final JND is defined as the dominant effect between activity masking and luminance adaptation. The drawback of this model resides in the fact that it does not distinguish between edge and texture regions. In [11] Yang et al. proposed an improved model that takes into account the luminance masking of Chou and $\mathrm{Li}$ and modifies the texture masking by introducing a factor based on Canny?s edge detector. The final masking model is an additive combination of the two maskings. However, in the literature, there are no results which validate such an idea [12]. Qi et al., in [7], proposed to use Chou and Li's JND, which only considers the first three rules summarized by Barni. However, they used the final masking proposed by Chou as their luminance masking. Spatial domain methods are simple and efficient, but the final rule 
chosen to combine all the possible effects observed in the HVS is often not well justified. In addition, Barni's Rule 4 is not usually taken into account. For these reasons, some prefer to design JND models in transform domains. Watson et al. [13] developed one of the first JND models in the Discrete Cosine Transform (DCT) domain. This model estimates the perceptibility changes in each DCT block. This block-based approach causes the loss of correlation between blocks [4]. Barni and Bartolini [14] [14] proposed a perceptual watermarking in the Discrete Wavelet Transform (DWT) domain. The weakness of the method comes from the so-called perceptual weighting of 3 terms which is far from being well-justified. Finally, Nguyen et al. [12] proposed a JND model in the DWT domain using a pyramidal representation. It incorporates three functions: contrast sensitivity, luminance adaptation and contrast masking, but does not include Barni's Rule 4. In this paper, a new perceptual image watermarking method is proposed which combines the advantages of both the wavelet domain and the spatial domain using a mixed-scale representation. Wavelet transformation allows to perform an image multiscale analysis. Mixed-scale representation makes that all scales are present in the spatial domain in only a single image. Wavelets coefficients high values correspond to a high grey level variations in the image and are called dominant coefficients. A high density of the dominant coefficients indicate high activity regions, i.e. textures. At the opposite, a low density of dominant coefficients correspond to uniform regions. Contours correspond to an intermediate situation between textures and uniform regions. Uniform, edge and textured areas are differentiated based on the density of the dominant wavelet coefficients. To choose between the three regions (flat, texture and contour) we use the FaberSchauder?s wavelet lifting scheme transform in which the wavelet coefficients are represented in a mixed-scale representation [15]. This takes advantage of the strong point of wavelet analysis (i.e. multiscale analysis) as well as the local properties of the image. Unlike the majority of JND models that let the method decides the type of JND to use through unclear rules, our method offers the possibility of choosing the optimal mask adapted to the pixel location by considering luminance masking and the edge masking developed by $\mathrm{Chou}$ and $\mathrm{Li}$, and In textured regions, we select the masking developed by Qi et al.. In order to take into account Barni's Rule 4, we also introduce an orientation factor [16]. The proposed method was tested on several natural images and compared to other perceptual models for watermarking purposes.

This paper is organized as follows: Section 2 presents the JND of Chou and Li and Qi et al. In section 3 we describe our watermarking method with JND using Faber-Schauder's mixed-scale wavelet lifting scheme. In section 4, we present the results obtained for the evaluation of visual quality and robustness on several natural images. Section 5 concludes the paper and provides some perspectives.

\section{PERCEPTUAL MODELS PROPOSED BY CHOU AND LI, AND QI ET AL}

\subsection{Luminance masking of Chou and $\mathrm{Li}$}

Chou and $\mathrm{Li}[10]$ proposed a luminance masking wherein the visibility threshold due to the luminance of the background is the luminance masking $J N D_{L}(x, y)$. The relationship between noise sensitivity and background luminance is the result of a subjective test as shown in Fig. 1(a). Fig. 1(c) shows an example of the luminance masking for the Lena image.

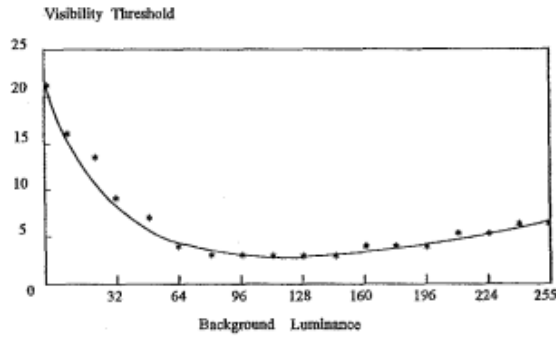

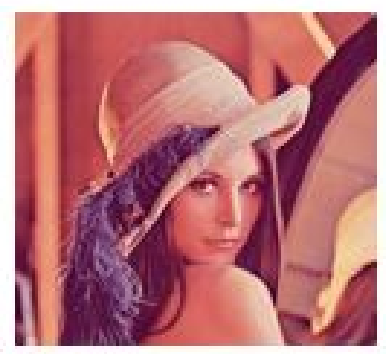

(b)

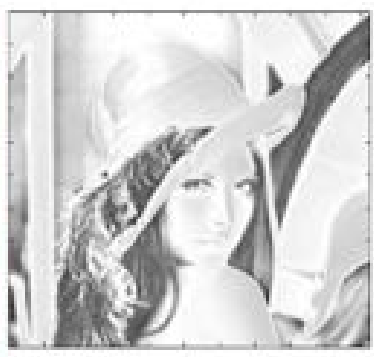

(c)
Fig. 1: (a) Visibility threshold relative to the background luminance modeled by Chou $[10]$. (b) Original Lena image. (c) Luminance masking $J N D_{L}(x, y)$ (with inverted color) obtained for the Lena image. Black parts in the masking correspond to high watermark embedding strength.

The luminance masking is the following for 8-bit gray scale images:

$$
J N D_{L}(x, y)=\left\{\begin{array}{c}
17\left(1-\left(\frac{b_{g}(x, y)}{127}\right)^{\frac{1}{2}}\right)+3 \text { for } b_{g} \leq 127 \\
\frac{3}{128}\left(b_{g}(x, y)-127\right)+3 \quad \text { for } \quad b_{g} \geq 127
\end{array}\right.
$$

$$
\begin{gathered}
b_{g}(x, y)=\frac{1}{32} \sum_{i=1}^{5} \sum_{j=1}^{5} I_{o}(x-3+i, y-3+j) . B(x, y), \\
B(i, j)=\left[\begin{array}{lllll}
1 & 1 & 1 & 1 & 1 \\
1 & 2 & 2 & 2 & 1 \\
1 & 2 & 0 & 2 & 1 \\
1 & 2 & 2 & 2 & 1 \\
1 & 1 & 1 & 1 & 1
\end{array}\right]
\end{gathered}
$$

In equation (2), $H$ and $W$ denote respectively the height and width of the image $I_{o} . b_{g}(x, y)$ is the average luminance of the background which is calculated by using the averaging filter B.

\subsection{Edge masking proposed by $\mathrm{Chou}$ and $\mathrm{Li}$}

The edge masking effect is studied by perceptual tests [10] such as that presented in Fig. 2. Chou and Li proposed to model this behavior by the linear function, $J N D_{E}(x, y)$ which reads as follows:

$$
\begin{gathered}
\left.J N D_{E}\left(b_{g}(x, y), m_{g}(x, y)\right)=m_{g}(x, y)\right) \cdot \alpha\left(b_{g}(x, y)\right)+\beta\left(b_{g}(x, y)\right) \\
\alpha\left(b_{g}(x, y)\right)=b_{g}(x, y) \cdot 0.0001+0.115 \\
\beta\left(b_{g}(x, y)=\frac{1}{2}-b_{g}(x, y) \cdot 0.01\right. \\
m_{g}(x, y)=\max \left\{\left|\operatorname{grad}_{k}(x, y)\right|\right\} ; \quad k=1,2,3,4
\end{gathered}
$$


$\operatorname{grad}_{k}(x, y)=\frac{1}{16} \sum_{i=1}^{5} \sum_{j=1}^{5} I(x-3+i, y-3+j) \cdot G_{k}(x, y)$

where $b_{g}$ and $m_{g}$ are the average background luminance and the maximum weighted average luminance differences surrounding the pixel at position, respectively. $G_{k}(x, y)$ for $0<k<5$ is an operator to calculate the average luminance variations in four directions:

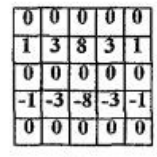

$G_{t}$

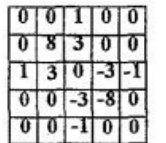

$G_{2}$

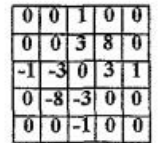

G,

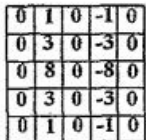

G.

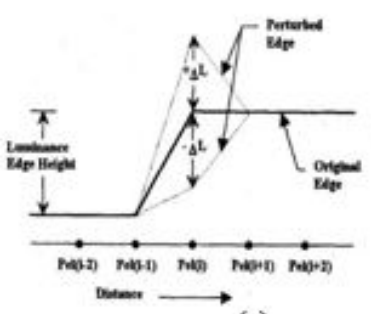

(a)

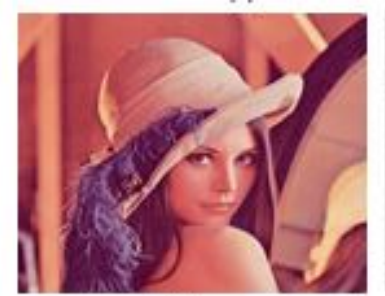

(c)

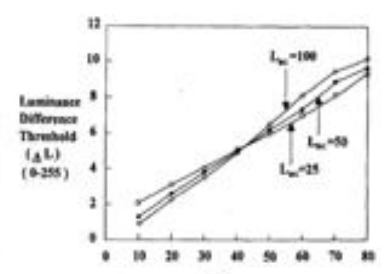

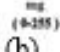

(b)

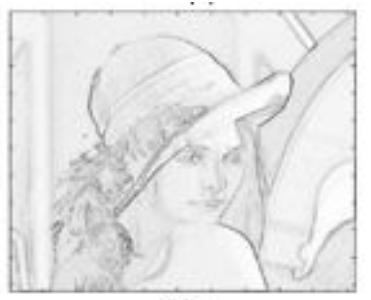

(d)
Fig. 2: (a) Stimuli to determine the visibility thresholds due to luminance changes. (b) Difference visibility thresholds for different background luminances [10]. (c) Lena image. (d) Edge Masking $J N D_{E}\left(I_{o}\right)$ (with inverted colors).

\subsection{Texture masking of Qi et al.}

For texture masking, we used the model proposed by Qi et al. [7]. The texture masking in Fig.3 (b) uses the absolute value of the distance between each pixel and the local average value of the pixels within a sliding window of L L pixels, as demonstrated in Eq. (4):

$$
\begin{gathered}
J N D_{T}\left(I_{o}\right)=\left|I_{o}(i, j)-\bar{I}_{o}(i, j)\right| \\
\left|\bar{I}_{o}(i, j)\right|=\frac{1}{(2 L+1)^{2}} \sum_{k=-L}^{L} \sum_{l=-L}^{L} I_{o}(i+k, j+l)
\end{gathered}
$$

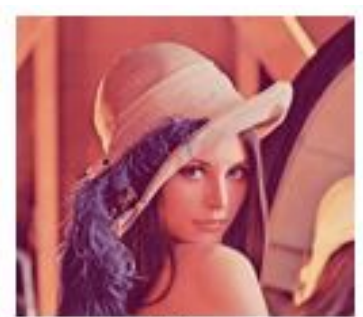

(a)

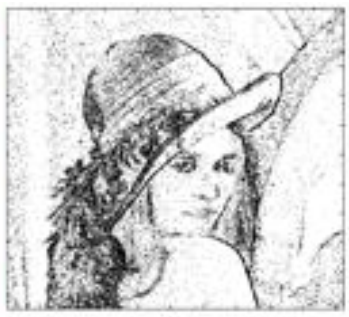

(b)
Fig. 3: (a) Lena image. (b)Texture Masking $J N D_{T}\left(I_{o}\right)$ with sliding window $3 \times 3$ (with inverted colors)

\section{PERCEPTUAL MASKING BASED ON MIXED-SCALE WAVELET}

We propose a new perceptual image watermarking method that combines the advantages of both the wavelet domain and the spatial domain. A mixed-scale wavelet representation is applied. Uniform, edge and textured areas are differentiated based on the density of the dominant wavelet coefficients [15]. This makes the choice between the JNDs more efficient by choosing the masking according to the pixel location. The following scheme (Fig. 4) presents the proposed perceptual mask, where the JNDs presented in the previous section are used. By discriminating the local texture in three categories, the most appropriate JND model can be applied in order to provide a more adapted JND model. Luminance masking developed by Chou and $\mathrm{Li}$ is applied in case of uniform areas. The masking developed by Qi et al.is preferred in textured regions, as the one developed by Chou and $\mathrm{Li}$ for edge areas.

In order to take into account Barni's Rule 4 we also introduce an orientation factor: for orientation at 45, the texture and edge masking are multiplied by $\sqrt{2}[16 \mid$. The orientation at each pixel is determined as the maximum energy given using the filters G1 to G4 presented in section 2.2 to estimate the average luminance variations in four directions.

\subsection{The texture discrimination based on mixed-scale representation}

As Mallat shows in his works on wavelets, wavelet transform can be considered as a multi-scale Canny or Marr-Hildrith contour detector [17]. Our method is based firstly on a thresholding to determine dominant coefficients which corresponds to high activity in the image, and secondly point out uniform, contour or textured regions based on the density values of dominant coefficients. Among all the possible type of wavelet transforms, we chose the wavelet transform of Faber-Schauder (FSDWT) [18] which is a good compromise for region discrimination (uniform, contour, and texture regions) as explained in the following.

First, let us consider the case of uniform regions. Haar wavelet transform can only handle strictly uniform regions. Faber-Schauder wavelet transform includes, in addition, linear regions, while other more sophisticated WT (Daubechies or higher order lifting schemes for example) include complex situations for uniform regions. Concerning contours, Haar only considers step contours, FS includes in addition ramp contours, and sophisticated wavelets more contours possibilities. Finally, texture are complex patterns where possible contours of various shape and spatial repetitions occur. As a conclusion, we privilege the FS wavelets because the Haar one is too rigid to face real world situations, while sophisti- 
cated WT are not selective enough when a choice has to be made between uniform, contour or textures. More details are presented in [18].

The Faber-Schauder wavelet transform (FSWT) and inverse transform (IFSWT) can be given by a simple arithmetic lifting scheme that allows an exact calculation of the wavelet coefficients [18] Eq.(5) and Eq.(6):

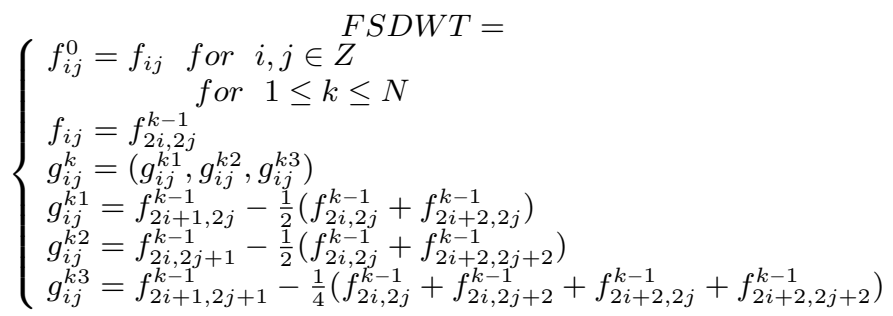

$$
\begin{gathered}
\text { for } 0 \leq k \leq N-1 \text { SWWT }= \\
\left\{\begin{array}{c}
f_{2 i, 2 j}^{k}=f_{i j}^{k} \\
f_{2 i+1,2 j}^{k}=g_{i j}^{k+1,1}+\frac{1}{2}\left(f_{i, j}^{k+11}+f_{i+1, j}^{k+1}\right) \\
f_{2 i, 2 j+1}^{k}=g_{i j}^{k+1,2}+\frac{1}{2}\left(f_{i, j}^{k+1}+f_{i, j+1}^{k+1}\right) \\
f_{2 i+1,2 j+1}^{k}=g_{i j}^{k+1,3}+\frac{1}{4}\left(f_{i, j}^{k+1}+f_{i+1, j}^{k+1}+f_{i, j+1}^{k+1}+f_{i+1, j+1}^{k+1}\right)
\end{array}\right.
\end{gathered}
$$

Where $\left(f_{i j}^{0}\right)$ represent the image to transform, $\left(f_{i j}^{k}\right)$ and $\left(g_{i j}^{k}\right)$ represents approximation and details wavelet coefficient at scale $k$.. Usually a pyramidal representation is used to show the result of the wavelet transformation as illustrated in Fig. 5(a). In the mixedscale representation, each wavelet coefficient is positioned at the place where its associated wavelet function is localized. Only one transformed image results (instead of pyramidal images) having the same size as the original image. It points out textured and edge areas in a given image [18 19] as illustrated in Fig. 5(b). A close look shows that the concentration of dominant coefficients is higher in textured regions than in those which are contours. In flat regions, very few dominant coefficients are present. Hence, in the mixedscale representation, the density of the dominant coefficients can be used as a discriminator between uniform, textured and edge areas [18 [19].

The proposed approach for the discriminator is as follows. First we transform the image using the mixed-scale Faber-Schauder lifting scheme. We select the $10 \%$ highest nonzero coefficients to be the dominant ones, as suggested by S. Mallat [20] for natural images. For each pixel we determine the number of dominant coefficients observed in a 33 windows centered on the pixel. For most natural images, when the sliding window is positioned in the middle of a uniform region (perfectly flat or linear region), 0 or 1 dominant coefficient is present in the window. When the window is centered on a strong edge (step or ramp in horizontal, vertical or diagonal directions), the number of dominant coefficient is high with a maximum of 6 .

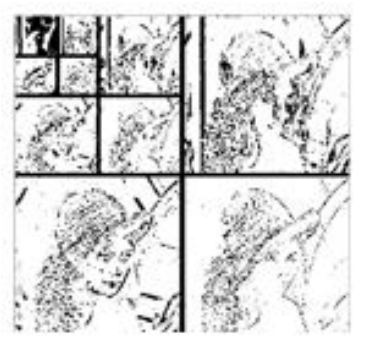

(a)

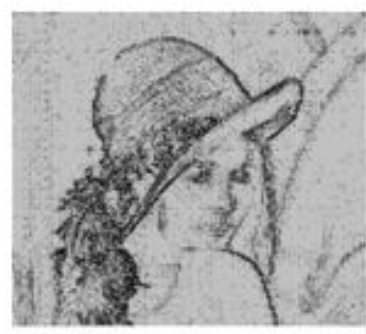

(b)
Fig. 5: (a) Pyramidal wavelet scale representation. (b) mixed-scale representation.

The case of textures is more complex and does not permit the simple analysis performed in the case of a flat or contour regions. What can be said is that the number of dominant coefficients is high and that the maximum value of dominant coefficient can be up to 9 . We decide to define the different regions in the image as well as the related JND?s to apply by the number of dominant coefficients in the $3 \times 3$ window: i) from 0 to 2 dominant coefficients the region is considered as uniform and a the Chou luminance JND is applied, ii) 3 and 4 dominant coefficients corresponds to a contour region and Chou edge JND is chosen, iii) else the region is textured and the Qi texture JND is applied. These numbers of dominant coefficients were chosen from experimental results conducted on a large set of natural images. The number of dominant coefficients in the window corresponds, by dividing by 9 , to a subjective notion of density of dominant coefficients and can be attached to the notion of region activity as defined by Chou and $\mathrm{Li}$.

As observed in the figure 6(b) for the synthetic image of 6(a), the various regions (uniform, contour and texture) are present at the place where we expect them to be. In figure 7(a), the same analysis is presented for the Lena image as well as the final masking we propose.

To synthetize, the processing is the following:

\section{-First step: Applying the Faber-Schauder transform $I_{F S D W T}=F S D W T\left(I_{o}\right)$}

- Second step: Find the dominant coefficients $c_{d}$

A coefficient is dominant (significant) if its absolute value exceeds a threshold which is fixed to $S_{c}=10 \%$

-Third step: Find the density d of the dominant coefficients.

$d=\frac{c_{d}}{N_{c_{d}}}$

With $N_{c_{d}}$ is the number of dominant coefficients in a bloc 3 by 3.

-Fourth step: choose the optimal masking using the mixed-scale wavelet transform. 


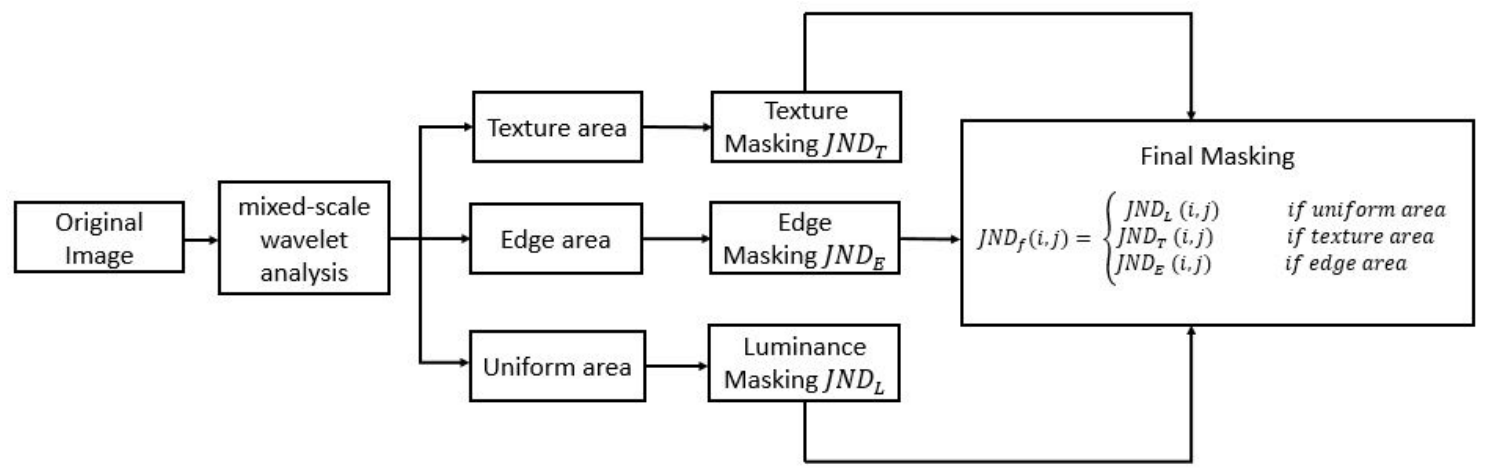

Fig. 4: Final Masking using the mixed-scale wavelet transform
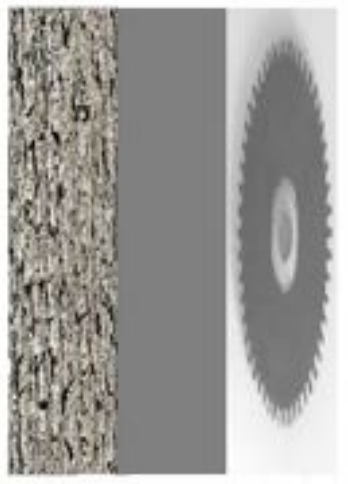

(a)

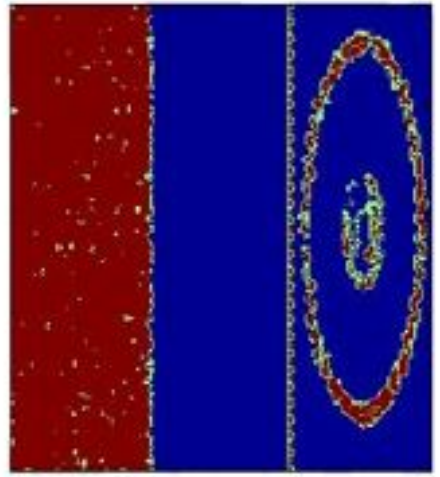

(b)
Fig. 6: (a) a synthetic image composed of uniform regions, edges and textures. In (b) the blue color corresponds to uniform regions with a number of dominant coefficients from 0 to 2 , the green color corresponds to edge regions with a number of dominant coefficients from 3 to 4 and the red color corresponds to the textured regions with a number of dominant coefficients from 5 to 9 .

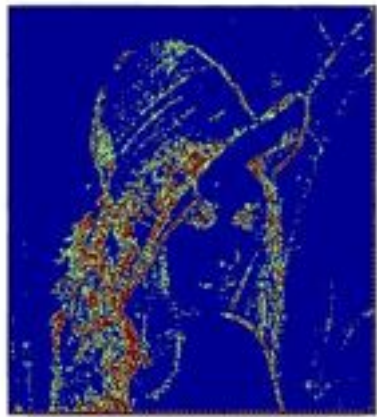

(a)

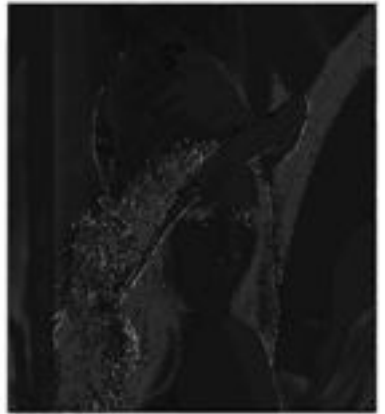

(b)
Fig. 7: (a) Lena image where the blue color corresponds to uniform regions, the green color corresponds to edge regions and the red color corresponds to the textured regions. (b) final masking using the JND models of Chou and Li and Qi et al. and the mixed scale FSWT selector.

\section{RESULTS}

We compared the performances of the proposed mixed-scale wavelet watermarking to four other state-of-the-art JND based watermarking methods:

(1) Chou and Li's watermarking [10],

(2) Qi et al.'s spatial watermarking [7],

(3) Barni et al.'s DWT watermarking [16],

(4) Watson's DCT-based watermarking [13].

These methods were selected according to their popularity and efficiency. If they have been adapted in several more recent works, the concepts behind the new proposals are very similar. For example, Watson's method remains the reference approach to define DCT masks as well as Barni rules for defining psychovisual masks.

Our proposal is based on the original concepts provided in the pioneer methods. These concepts are intelligently embedded to provide a new scheme. For this reason, they have been selected to perform the comparisons with our proposal.

We used a set of 50 natural color images of variable textures and edges as shown in Fig. 8. For all the methods, only the luminance was watermarked (chrominance components were not modified). In our method, the watermark was inserted in the spatial domain with a spread spectrum technique used for watermarking as described in [21] in our case, we use a sequence of 64 bits. The spread sequence is modulated with a binary pseudo-random sequence $W \in\{-1,1\}$ to obtain the watermark. The quality of the watermarked images was assessed, and the robustness to attacks was evaluated, as detailed in the following sections.

\subsection{Visual quality of watermarked images}

We first evaluated the visual quality of the watermarked image, using two types of metrics:

-objective metrics such as Weighted Peak Signal-to-Noise Ratio (WPSNR) and the Structural SIMilarity (SSIM),

- a subjective metric by conducting subjective tests with human observers.

The first objective metric used was the Weighted Peak Signal-toNoise Ratio (WPSNR) proposed in [22]. Just like PSNR, a high value of WPSNR indicates that the image is less distorted. Values above $40 \mathrm{~dB}$ indicate almost invisible degradation and values below 
$30 \mathrm{~dB}$ indicate high degradation [23]. For an 8-bit grayscale image, the WPSNR is as follows:

$$
W P S N R=10 \log _{10} \frac{\max \left(I_{o}\right)^{2}}{\left\|\left(I_{w}-I_{o}\right) \cdot N V F\right\|^{2}}
$$

with

$$
N V F=\frac{1}{1+\theta \cdot \sigma_{I_{o}}^{2}(i, j)}
$$

where $\sigma_{I_{o}}^{2}(i, j)$ denotes the local variance of the image in a window centered on the pixel with coordinates $(i, j)$ and $\theta$ is a tuning parameter which plays the role of the contrast adjustment. We also used the Structural SIMilarity (SSIM) proposed by [24] to test the visual quality (a SSIM close to 1 indicates a good visual quality):

$$
S S I M=\frac{1}{W} \sum_{i=1}^{W} l(i) c(i) s(i)
$$

where

$$
\begin{gathered}
l(i)=\frac{\overline{2 x(l)} \cdot \overline{y(l)}+C_{1}}{\left.\overline{x(l)})^{2}+\overline{y(l)}\right)^{2}+C_{1}} ; c(i)=\frac{2 \sigma_{x}(i) \cdot \sigma_{y}(i)+C_{2}}{\sigma_{x}^{2}(i) \cdot \sigma_{y}^{2}(i)+C_{2}} \\
s(i)=\frac{2 \sigma_{x y}(i)+C_{3}}{\sigma_{x}(i) \cdot \sigma_{y}(i)+C_{3}} \\
C_{1}=\left(L \cdot K_{1}\right)^{2}, C_{2}=\left(L \cdot K_{2}\right)^{2}, C_{3}=\left(L \cdot K_{3}\right)^{2}
\end{gathered}
$$

and where $\mathrm{L}$ is the dynamic of the grayscale. $K_{1}$ ) and $K_{2}$ ) are two constants fixed by the authors at 0.01 and 0.03 , respectively. $\sigma_{x}$ and $\sigma_{y}$ can be viewed as estimates of the contrast of $x$ and $y$.

For the subjective metric fifteen subjects were recruited for the tests [25]. During the experiments, tested images appeared randomly. Images were presented on a screen in standardized conditions and the viewing distance was set at 6 times the image height. The grading scale consisted of MOS (Mean Opinion Scale) from 1 to 5 . For the set of the 50 images tested, experimental results are reported in Table 1 for the two objective metrics and in Table 2 for the subjective metric.

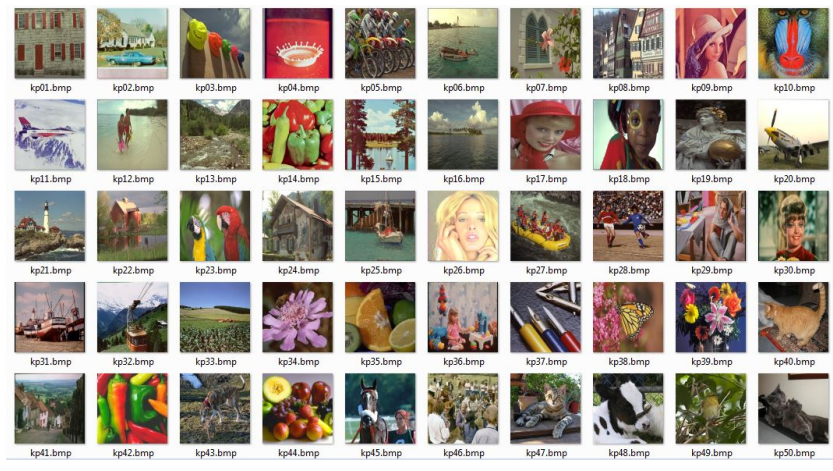

Fig. 8: 50 test images used to evaluate performances of the proposed JND model.

It can be seen that Barni's method yields the best perceptual quality evaluated by means of objective tests. Except for the Watson method, the 3 others are also of high quality. Concerning the subjective tests, the Barni's method is a little better than the proposed one, while others are not so efficient. In conclusion, Barni's method offers the best visual quality, our method is very close to Barni's one, while the 3 others are less powerful.

\subsection{Robustness to attacks}

Second, simulated attacks were performed to test the robustness of the watermark as shown in Fig. 9. We calculated the Bit-Error-Rate (BER) of the extracted message.

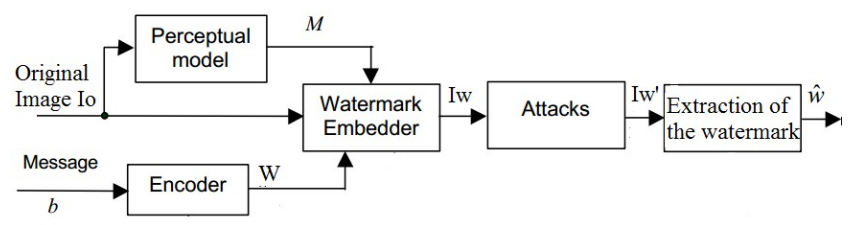

Fig. 9: Scheme of a watermarking test process using perceptual models.

In order to test the robustness of the proposed method, we performed different types of signal and geometric attacks, namely: JPEG compression. Rotation, Scaling and Croping. Gaussian noise addition (AWGN), Denoising attack: low pass filtering and median filtering. Histogram equalization and Contrast adjustment. Results are presented in Table 3. We use the BER to measure the rate of watermark extraction. BER is defined as the ratio of number of erroneous watermark bits extracted to the total number of embedded bits. Low values of BER mean that the watermarking method results have a good robustness.

The results of watermarked images after JPEG compression with different quality factor $(\mathrm{QF}=80 \%, 40 \%$ and $10 \%)$, did not exceed 0.2 even under the situation of compression ratio $10 \%$. The DCT method results are still far from the spatial one.

For the rotation attack the watermarked images are rotated by $30^{\circ}$, $45^{\circ}$ and $90^{\circ}$. For $30^{\circ}$ the message was extracted. All methods used have a low robustness against the rotation attack after 45 . The watermarked images is cropped $3 \%, 5 \%$ and $7 \%$ at bottom left corner (BL). The attacked image was scale time the size 0.2 and 0.5 of original image. Performances between the methods are comparable.

For noise attacks: the watermarked images are subjected to AWGN (with mean zero and variance $=0.1,0.2$ and 0.5 ). The results of BER for the two tested JND spatial methods [7 [10] are under our method with a good robustness even with high corruption and are far better than the DCT method [13].

The watermarked images are filtered with filter of size $3 \times 3,5 \times 5$ and $7 \times 7$. Even with a high filter size the watermark are extracted for the proposed method and the three other JND models except for the DCT one.

Finally the watermark extracted after histogram equalization and contrast adjustment respectively, are equal to zero for all images watermark for the three tested JND spatial methods and the proposed method. JND spatial methods still far better than the DCT method.

From the results above, we can see that our method is quite robust against attack and gives better result compared to the spatial one. Throughout these results, it is clear that using perceptual models helps to improve not only transparency but also robustness of watermarking.

As a final conclusion, the proposed method is the best trade-off between visual quality and resistance to attacks among the tested methods. In (fig 10), the extracted message after some attacks on the image mandrill is depicted as well as the Ber value. 
Table 1.: Perceptual quality evaluation by means of 2 objective metrics (WPSNR and SSIM).

\begin{tabular}{|c|c|c|c|c|c|}
\hline Objective quality metrics & $\begin{array}{c}\text { JND of Chou } \\
{[10]}\end{array}$ & $\begin{array}{c}\text { JND of Qi } \\
{[7]}\end{array}$ & $\begin{array}{c}\text { JND of Barni } \\
{[16]}\end{array}$ & $\begin{array}{c}\text { JND of Watson } \\
{[13]}\end{array}$ & $\begin{array}{c}\text { Proposed } \\
\text { Method }\end{array}$ \\
\hline $\begin{array}{c}\text { Averaged WPSNR (dB) of 50 } \\
\text { images }\end{array}$ & 34.35 & 34.35 & 34.89 & 31.56 & 34.41 \\
\hline Averaged SSIM of 50 images & 0.81 & 0.81 & 0.86 & 0.74 & 0.84 \\
\hline
\end{tabular}

Table 2.: Perceptual quality evaluation by means of a subjective metric.

\begin{tabular}{|c|c|c|c|c|c|}
\hline Objective quality metrics & $\begin{array}{c}\text { JND of Chou } \\
{[10]}\end{array}$ & $\begin{array}{c}\text { JND of Qi } \\
{[7]}\end{array}$ & $\begin{array}{c}\text { JND of Barni } \\
{[16]}\end{array}$ & $\begin{array}{c}\text { JND of Watson } \\
{[13]}\end{array}$ & $\begin{array}{c}\text { Proposed } \\
\text { Method }\end{array}$ \\
\hline $\begin{array}{c}\text { Averaged WPSNR (dB) of 50 } \\
\text { images }\end{array}$ & 34.35 & 34.35 & 34.89 & 31.56 & 34.41 \\
\hline Averaged SSIM of 50 images & 0.81 & 0.81 & 0.86 & 0.74 & 0.84 \\
\hline
\end{tabular}

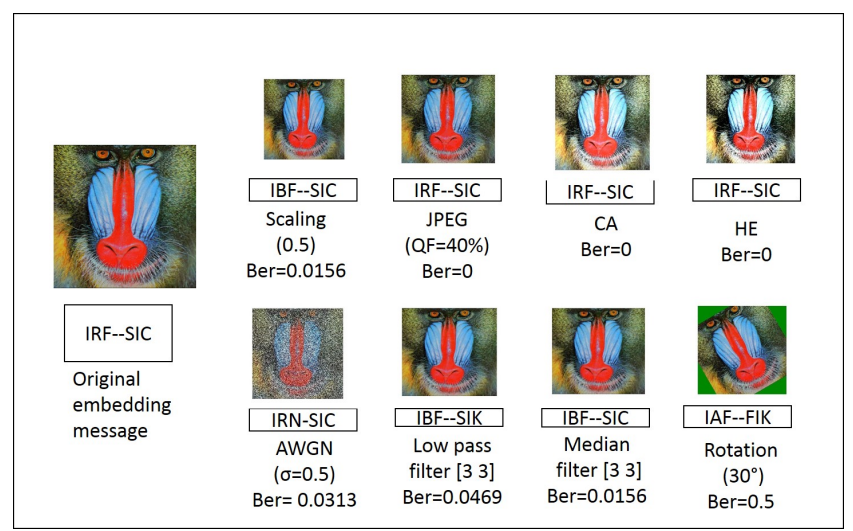

Fig. 10: The extracted message after some attacks on the image mandril.

\section{CONCLUSION}

In this paper, we have proposed a new watermarking method based on a mixed-scale Faber-Schauder wavelet lifting scheme. The DNA of our method relies in its capacity to accurately discriminate uniform, contour and textured regions allowing to apply specific JND models considered as references in the watermarking community. It is generic in the sense where any specific masks can be embedded. Our algorithm chooses the optimal mask adapted for each of the regions and takes into account Barni's Rule 4 concerning the angular sensitivity of the HVS to local activity. We tested both the visual quality and the robustness to attacks of the proposed watermarking method and compared it to four other state-of-the-art methods. Results show that the proposed method yields a high visual quality. In addition, our method is very robust against attacks compared to spatial JND models. In synthesis, Barni?s method offers the best visual quality among the methods. However our method is very similar in term of efficiency while being highly more resistant to most of attacks. This last point is of great importance for real applications. The proposed method is the best trade-off between visual quality and resistance to attacks among the 5 tested methods. In order to improve the robustness of our method, we intend to take into account the modulation transfer function of the HVS concerning the textured area. We also intend to determine the threshold values that select the various regions according to image properties of the given image.

\section{REFERENCES}

[1] A. Beghdadi, M.C. Larabi, A. Bouzerdoum, and K.M. Iftekharuddin. 2013. A survey of perceptual image processing methods, Sig. Proc. Image Commun. 28(8), 811-831.

[2] R.B. Wolfgang, C.I. Podilchuk, and E.J. Delp. 1999. Perceptual watermarks for digital images and video, In: Proceedings of the IEEE, Special Issue on Identification and Protection of Multimedia Information, vol. 87, pp. 1108-1126

[3] M. Barni, and F. Bartolini. Boca Raton 2004. Watermarking Systems Engineering Enabling Digital Assets Security and Other Applications, CRC Press, ISBN: 0-8247-4806-9. 
Table 3.: Results of average BER over 50 images after different attacks.

\begin{tabular}{|c|c|c|c|c|c|c|}
\hline \multicolumn{2}{|c|}{} & \multicolumn{5}{c|}{ Averaged BER of 50 images } \\
\hline $\begin{array}{c}\text { Types of } \\
\text { attacks }\end{array}$ & Intensity & $\begin{array}{c}\text { JND of } \\
\text { Chou [10] }\end{array}$ & $\begin{array}{c}\text { JND of } \\
\text { Qi [7] }\end{array}$ & $\begin{array}{c}\text { JND of } \\
\text { Barni [16] }\end{array}$ & $\begin{array}{c}\text { JND of } \\
\text { Watson [13] }\end{array}$ & $\begin{array}{c}\text { Proposed } \\
\text { Method }\end{array}$ \\
\hline \multirow{2}{*}{ JPEG } & $Q=80 \%$ & 0 & 0 & 0.0031 & 0.3091 & 0 \\
\cline { 2 - 7 } & $\mathrm{Q}=10 \%$ & 0.1628 & 0.1628 & 0.1647 & 0.4306 & 0.1803 \\
\hline \multirow{2}{*}{ Rotation } & $30^{\circ}$ & 0.4878 & 0.4878 & 0.4878 & 0.4894 & 0.4869 \\
\cline { 2 - 7 } & $45^{\circ}$ & 0.5100 & 0.5100 & 0.5119 & 0.5034 & 0.5088 \\
\hline \multirow{2}{*}{ Croping } & $3 \%$ & 0.4866 & 0.4866 & 0.4847 & 0.4437 & 0.4884 \\
\cline { 2 - 7 } & $5 \%$ & 0.5094 & 0.5094 & 0.5075 & 0.5047 & 0.5069 \\
\hline \multirow{2}{*}{ Scaling } & 0.2 & 0.3844 & 0.3844 & 0.4156 & 0.5634 & 0.3825 \\
\cline { 2 - 7 } AWGN & 0.5 & 0.0553 & 0.0553 & 0.0425 & 0.5106 & 0.0450 \\
\cline { 2 - 7 } & $0=0.1$ & 0.0016 & 0.0016 & 0.0147 & 0.4656 & 0.0003 \\
\hline \multirow{2}{*}{ Low pass filter } & 0.5 & 0.0453 & 0.0478 & 0.0784 & 0.4753 & 0.0432 \\
\cline { 2 - 7 } & $3^{*} 3$ & 0.1047 & 0.1047 & 0.1072 & 0.5334 & 0.0950 \\
\hline \multirow{2}{*}{ Median filter } & $3^{* 3} 3$ & 0.0244 & 0.0244 & 0.0259 & 0.6184 & 0.0294 \\
\cline { 2 - 7 } & $7^{*} 7$ & 0.2491 & 0.2491 & 0.3141 & 0.5547 & 0.2344 \\
\hline $\begin{array}{c}\text { Histogram } \\
\text { equalization }\end{array}$ & & 0 & 0 & 0 & 0.4537 & 0 \\
\hline $\begin{array}{c}\text { Contrast } \\
\text { adjustment }\end{array}$ & & 0 & 0 & 0 & 0.3991 & 0 \\
\hline
\end{tabular}

[4] I. J. Cox, M. Miller, J. Bloom, J. Fridrich, and T. Kalker. 2007. Digital watermarking and steganography. Morgan Kaufmann.

[5] H. H. Tsai, H. C. Tseng, and Y. S. Lai. 2010. Robust lossless image watermarking based on trimmed mean algorithm and support vector machine, J. Syst. Softw. 83(6), 1015-1028.

[6] L. Li, X. Yuan, Z. Lu, and J. S. Pan. 2010. Rotation invariant watermark embedding based on scale adapted characteristic regions, Inf. Sci. 180(15), 2875-2888.

[7] H. Qi, D. Zheng, and J. Zhao. 2008. Human visual system based adaptive digital image watermarking, Sig. Process. 88(1), 174-188.

[8] P. Bas, J.M. Chassery, and B. Macq. 2002. Mthode de tatouage fond sur le contenu, Traitement du Signal. 19(1), 11?18.

[9] Y. Niu, M. Kyan, L. Ma, A. Beghdadi, and S. Krishnan. 2013. Visual saliency's modulatory effect on just noticeable distortion profile and its application in image watermarking, Sig. Process. Image Commun. 28(8), 917-928

[10] C. H. Chou, and Y. C. Li. 1995. A perceptually tuned subband image coder based on the measure of Just noticeable-distortion profile, IEEE Trans. Circ. Syst. Video Technol. 5(6), 467-476.

[11] X. Yang, W. Lin, Z. Lu, E. Ong, and S. Yao. 2005. Motioncompensated residue preprocessing in video coding based on just-noticeable-distortion profile, IEEE Trans. Circuits Syst. Video Technol. 15(6), 742-752.

[12] P. B. Nguyen, A. Beghdadi, and M. Luong,. 2013. Perceptual watermarking using a new Just-NoticeableDifference model, Sig. Proc. Image Commun. 28(10), 1506-1525.

[13] A. B. Watson. February 1993. DCT quantization matrices visually optimized for individual images, In: Proceedings of the SPIE Conference on Human Vision, Visual Processing and Digital Display IV, 1913, pp. 202-216.

[14] F. Bartolini, M. Barni, V. Cappellini, and A. Piva. October 1998. Mask building for perceptually hiding frequency embedded watermarks, in: Proceedings of the IEEE ICIP98 1450 454.

[15] M. Amar, R. Harba, H. Douzi, F. Ros, M. El Hajji, R. Riad and K. Gourrame. 2016. A JND Model Using a Texture-Edge Selector Based on Faber-Schauder Wavelet Lifting Scheme, In: Image and Signal Processing. Springer International Publishing, pp. 328-336.

[16] M. Barni, and F. Bartolini, Franco, and P. Alessandro. Improved wavelet-based watermarking through pixelwise masking. Image Processing, IEEE Transactions on, vol. 10, no 5, p. 783-791, 2001 
[17] J. Canny. 1986. A computational approach to edge detection. IEEE Trans.Patt. Anal.and Mach. Intell., 36:961-1005.

[18] H.Douzi, D. Mammass, and F. Nouboud. 2001. FaberSchauder wavelet transform, application to edge detection and image characterization, J. Math. Imag. Vis. 14(2), 91-101.

[19] M. El Hajji, H. Douzi, R. Harba, D. Mammass, and F. Ros. 2012. New image watermarking algorithm based on mixed scales wavelets, J. Electron. Imaging 21(1), 1-7.

[20] H. Douzi. 2001 Base d'ondelettes de Faber-Schauder et applications au traitement d'images, $\mathrm{PhD}$ thesis, University of Paris 6.

[21] F. Hartung, and B. Girod. October 1997 Fast Public-Key Watermarking of Compressed Video, Proc. of IEEE Int.Conf. Image Processing, vol. 1, pp-528-531.

[22] S. Voloshynovskiy, A. Herrigel, N. Baumgaertner, and T. Pun. January 2000. A stochastic approach to content adaptive digital image watermarking, In Information Hiding, Springer Berlin Heidelberg, pp. 211-236.

[23] A. Cheddad, J. Condell, K. Curran, and M. Kevitt. 2010. Digital image steganography suvey and analysis of current methods, Signal Process, Vol 90, pp. 727-752.

[24] Z. Wang, A. C. Bovik and E. P. Simoncelli. 2005. Structural approaches to image quality assessment, Handbook of image and video processing, 2nd Edition, Al Bovik, ed, Academic Press.

[25] J.L. Blin. 2003. SAMVIQ-Subjective assessment methodology for video quality, rapport technique BPN 056, EBU Project Group B/VIM Video Multimedia. 